\title{
Supporting Information for Electronic Absorption Spectra of Tetrapyrrole-based Pigments via TD-DFT: A Reduced Orbital Space Study
}

\author{
Kushal Shrestha, Kyle A. Virgil, and Elena Jakubikova*
}

Department of Chemistry, North Carolina State University, Raleigh, North Carolina 27695, USA.

\section{Table of Contents:}

Energy level diagram of orbital spaces

Figure S1

TD-DFT absorption spectra with all orbital spaces

Figure S2-S3. S2

Average errors in energies and intensities in all orbital spaces

Figure S4-S5.

Excitation density differences for RC with OS-100 and FOS

Figures S6-S8.

Transition density analyses of Q region excitations with OS-100 and FOS

Tables S1-S9,

Transition density analyses of Q region excitations with OS-60 and FOS

Figures S10-S19

\footnotetext{
*Corresponding author E-mail: ejakubi@ncsu.edu
} 


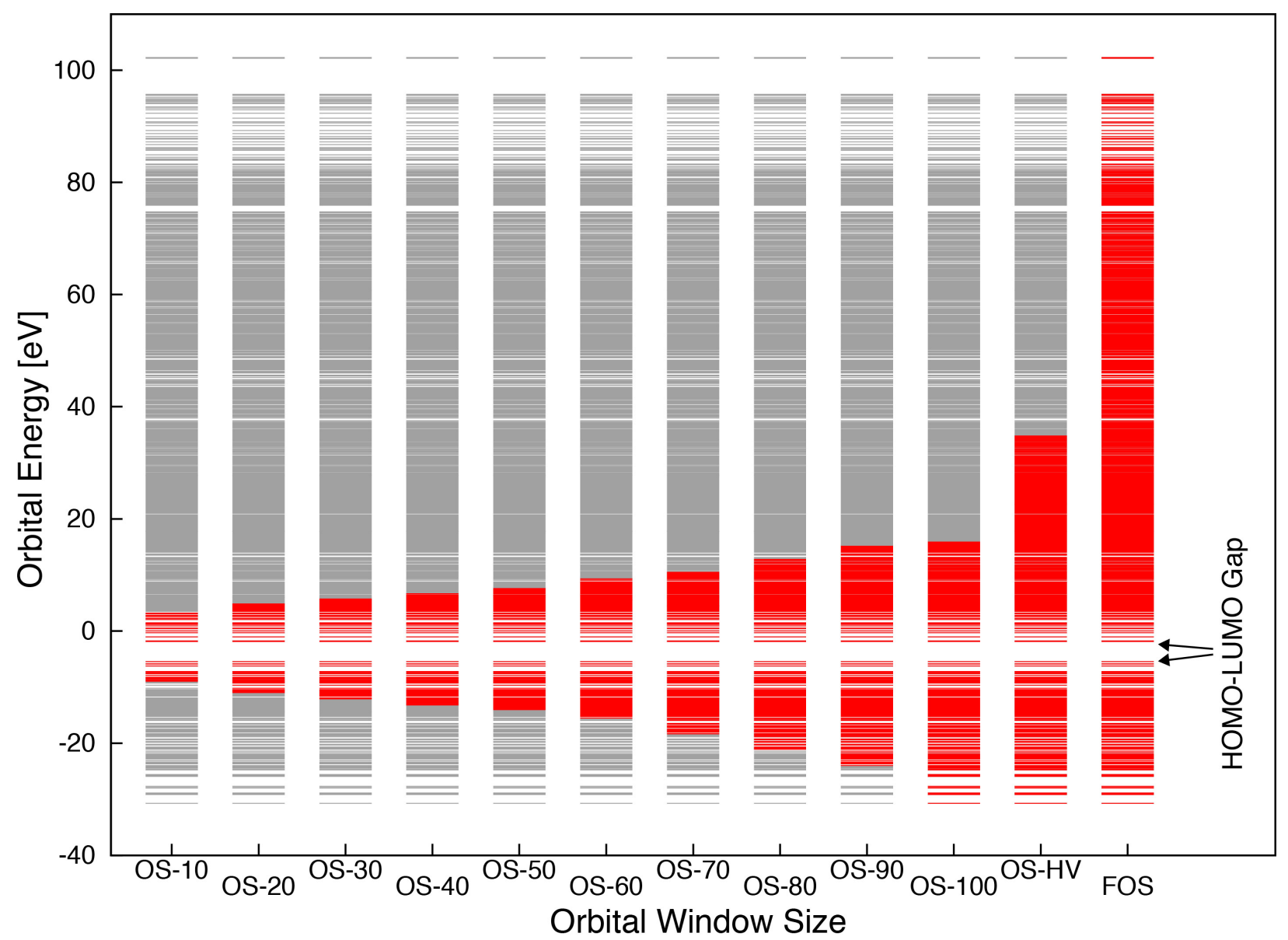

Figure S1. Orbital energy level diagrams for all orbital spaces employed in TD-DFT calculations for FbC-FbB. Only non-core orbital energy levels are shown. Energy levels corresponding to orbitals included in a given orbital space are highlighted in red. 


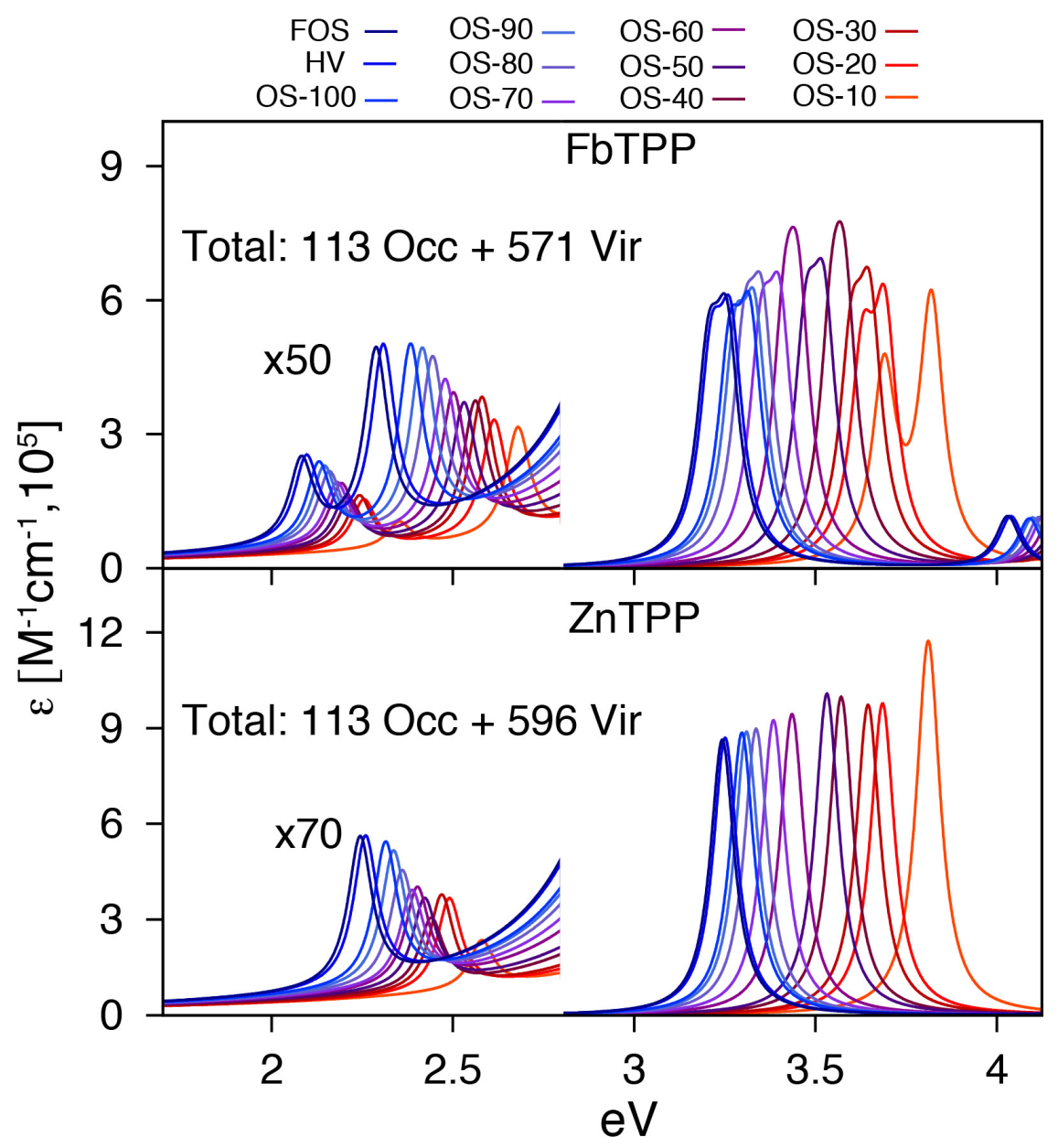

Figure S2. Calculated absorption spectra (20 excitations) for FbTPP (top panel) and ZnTPP (bottom panel) resulting from the full orbital space and smaller orbital spaces are shown. The orbital space size increases going from the red line ("OS-10" includes 10\% of non-core occupied orbitals and an equal number of virtual orbitals) to blue line ("FOS" includes all non-core occupied orbitals and all virtual orbitals). 


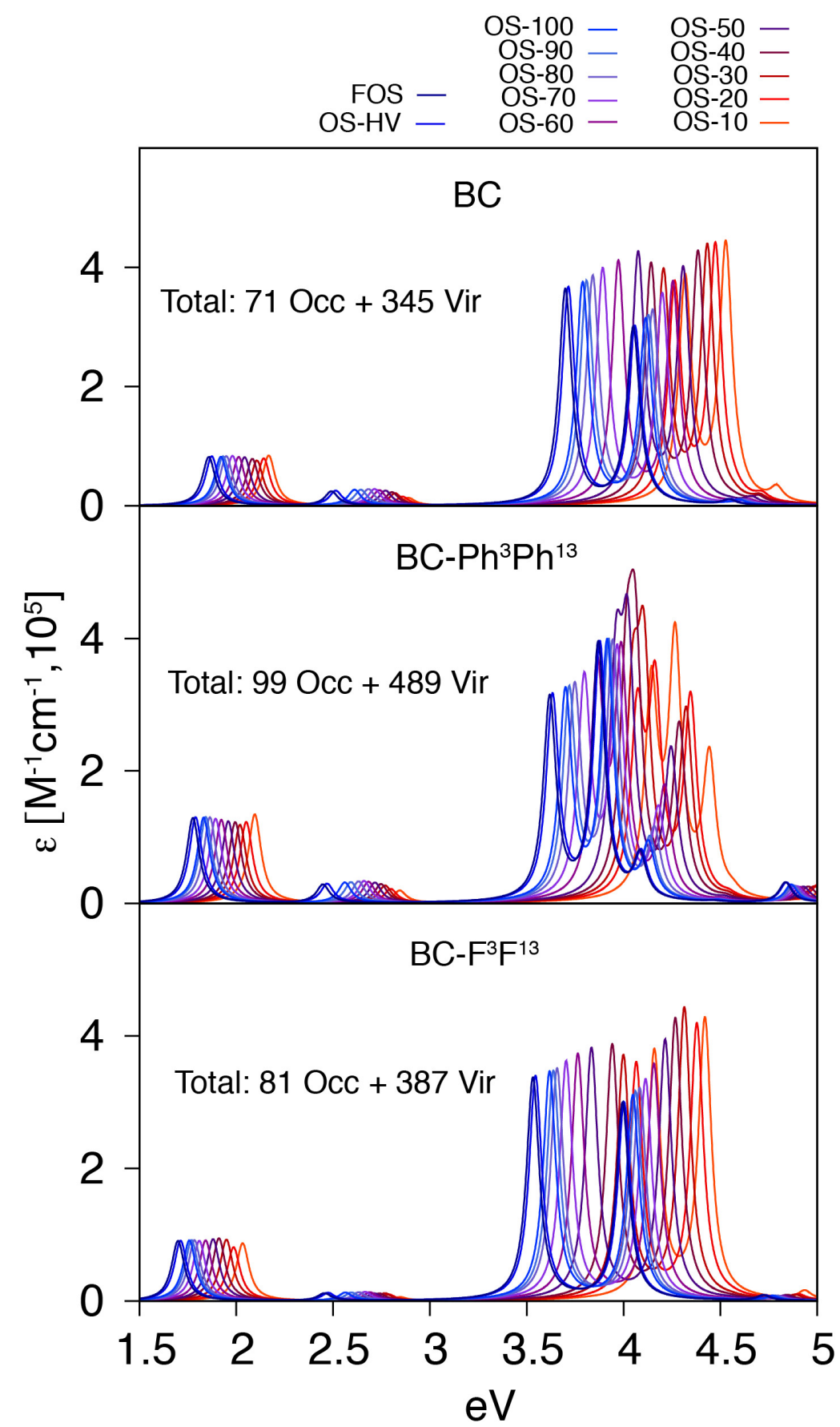

Figure S3. Calculated absorption spectra (20 excitations) for $\mathrm{BC}$ (top panel), BC-Ph3Ph13 (middle panel), $\mathrm{BC}-\mathrm{F}^{3} \mathrm{~F}^{13}$ (bottom panel) resulting from the full orbital space and smaller orbital spaces are shown. The orbital space size increases going from the red line ("OS-10" includes $10 \%$ of non-core occupied orbitals and an equal number of virtual orbitals) to blue line ("FOS" includes all non-core occupied orbitals and all virtual orbitals). 

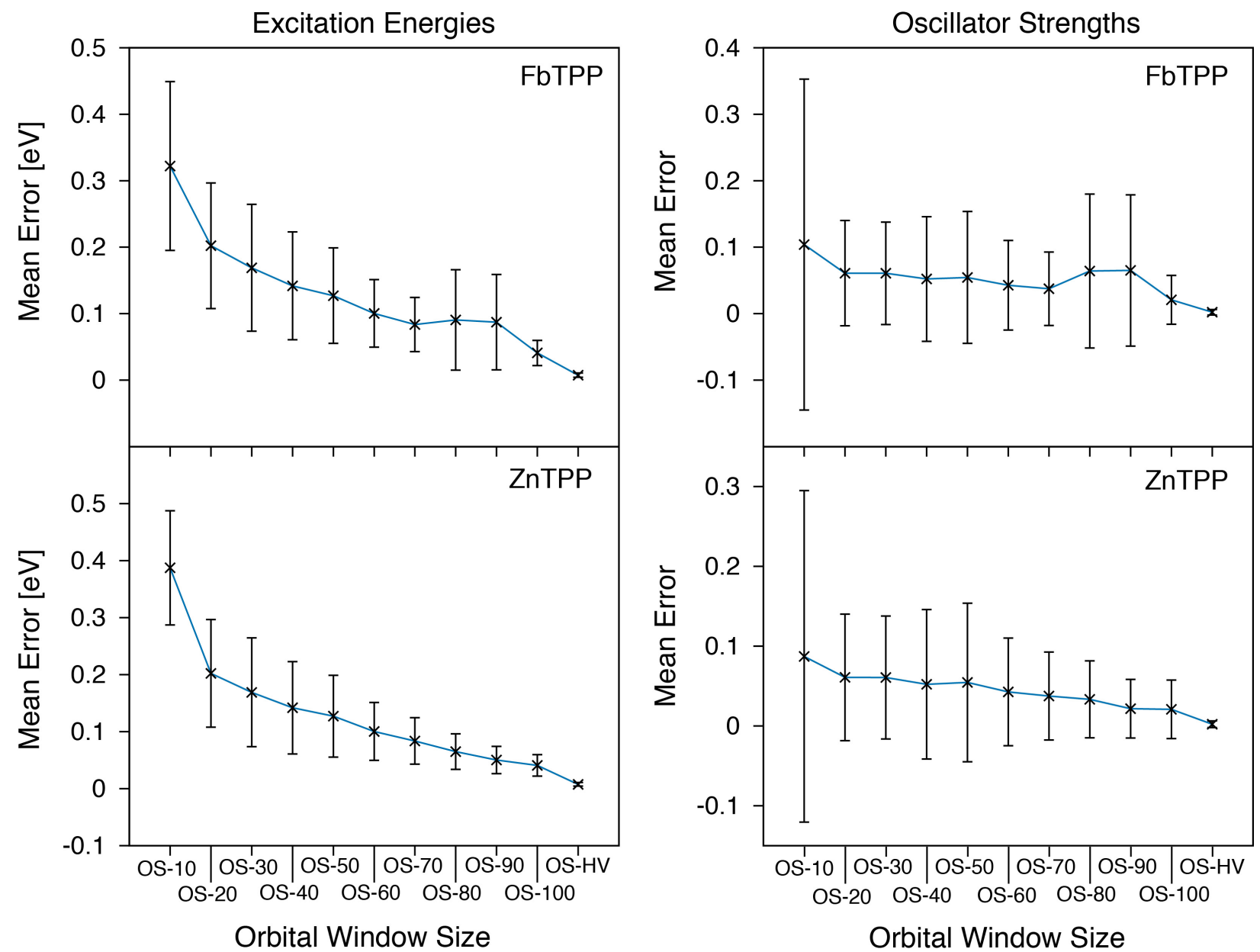

Figure S4. Average errors with standard deviations for the first 20 excitation energies and oscillator strengths of FbTPP and ZnTPP are plotted with respect to orbital space size. The errors shown are derived from comparison with values from the FOS TD-DFT results. 


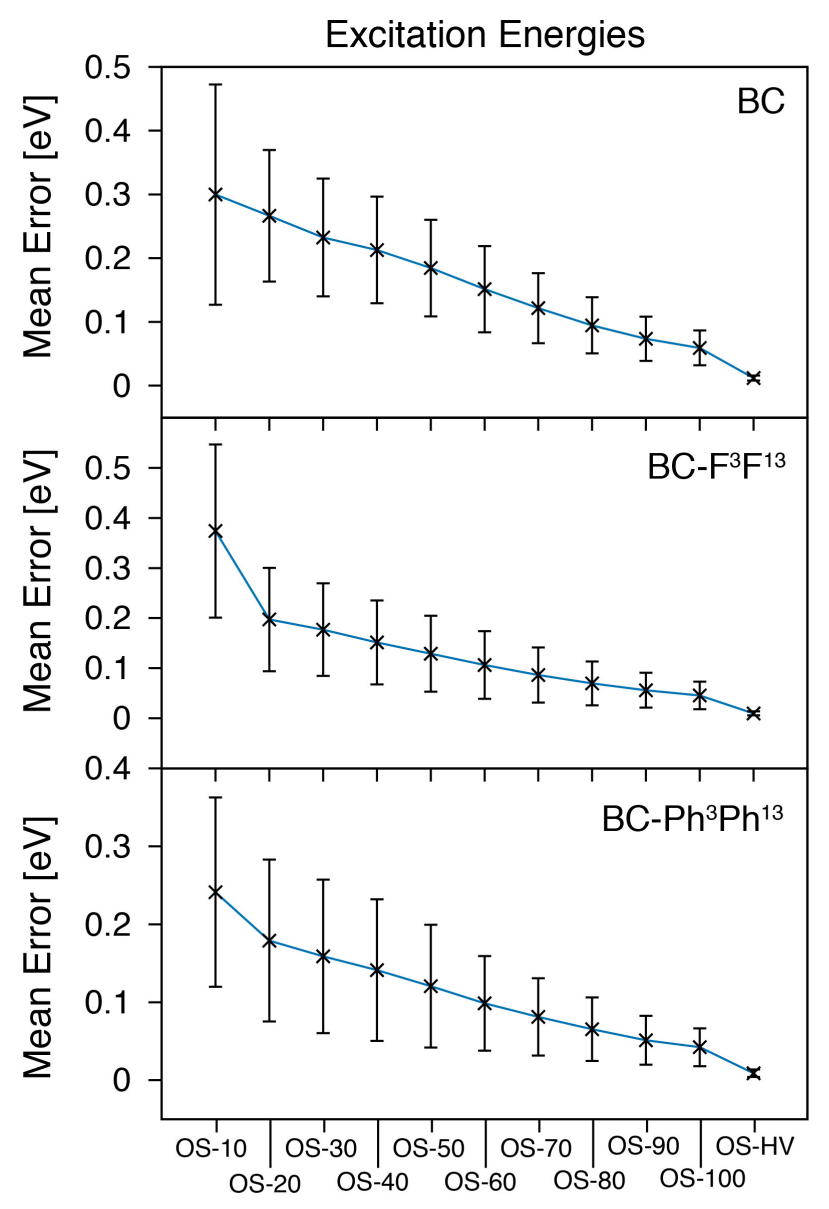

Orbital Window Size

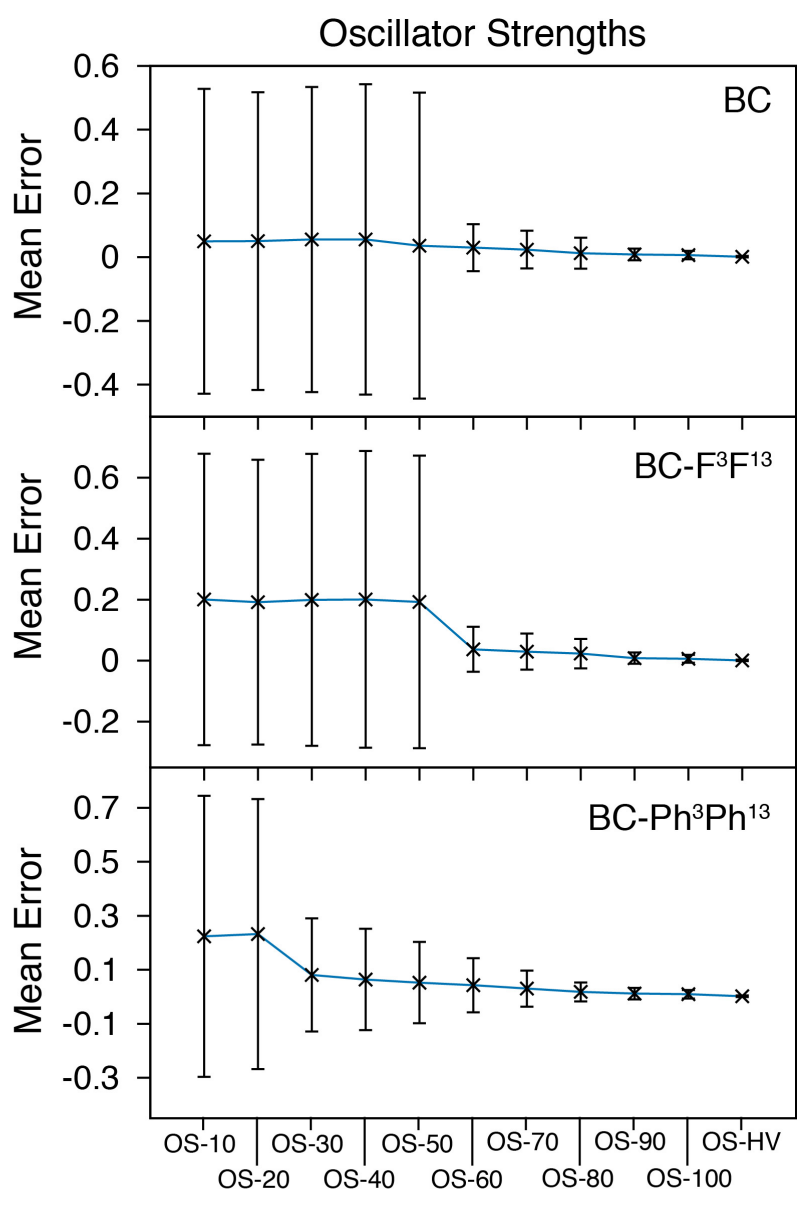

Orbital Window Size

Figure S5. Average errors with standard deviations for the first 20 excitation energies and oscillator strengths of $\mathrm{BC}, \mathrm{BC}-\mathrm{F}^{3} \mathrm{~F}^{13}$, and $\mathrm{BC}-\mathrm{Ph}^{3} \mathrm{Ph}^{13}$ are plotted with respect to orbital space size. The errors shown are derived from comparison with values from the FOS TD-DFT results. 


\section{Excitation Density Difference}

\section{Excitation 7:}

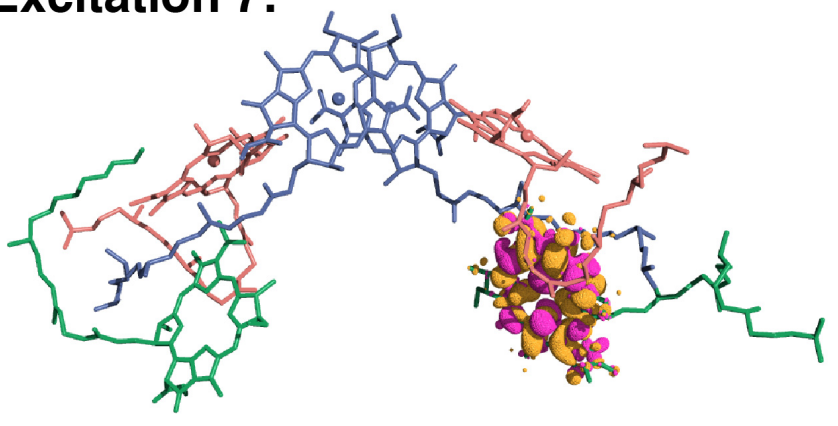

$\lambda=582.66 \mathrm{~nm}$, Localization: $\mathrm{H}_{\mathrm{L}}$

$$
\int \Delta \rho^{E X_{4}}(\overrightarrow{\mathbf{r}}) d \overrightarrow{\mathbf{r}}=+0.00103 e
$$

Figure S6. Positive (orange) and negative (pink) isosurfaces of the excitation density difference function, $\Delta \rho^{E X}(\vec{r})$, from FOS TD-DFT calculations for RC is shown. The seventh excitation is shown. All isosurfaces enclose $70 \%$ of the integrated change in $\Delta \rho^{E X}(\vec{r})$. 


\section{Excitation Density Differences}

OS-100

\section{Excitation 1:}

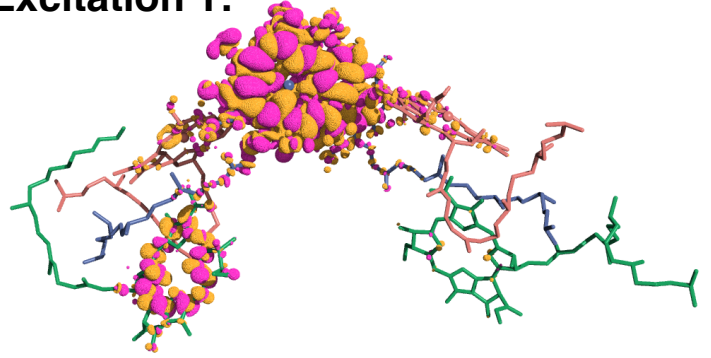

$\lambda=725.24 \mathrm{~nm}$, Localization: $\mathbf{P}$

$\int \Delta \rho^{E X_{1}}(\overrightarrow{\mathbf{r}}) d \overrightarrow{\mathbf{r}}=+0.00081 e$

\section{Excitation 2:}

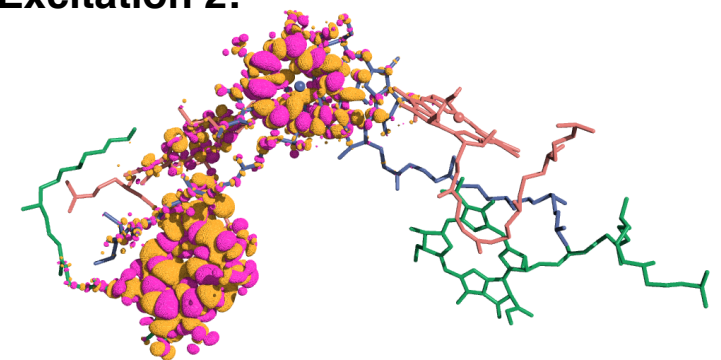

$\lambda=717.51 \mathrm{~nm}$, Localization: $\mathrm{H}_{\mathrm{M}}$

$\int \Delta \rho^{E X_{2}}(\overrightarrow{\mathbf{r}}) d \overrightarrow{\mathbf{r}}=+0.00087 e$

\section{Excitation 3:}

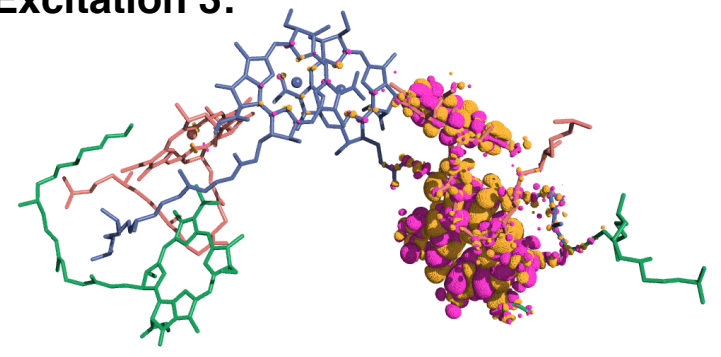

$\lambda=681.59 \mathrm{~nm}$, Localization: $\mathrm{H}_{\mathrm{L}}$

$\int \Delta \rho^{E X_{3}}(\overrightarrow{\mathbf{r}}) d \overrightarrow{\mathbf{r}}=+0.00084 e$

\section{FOS}

\section{Excitation 1:}

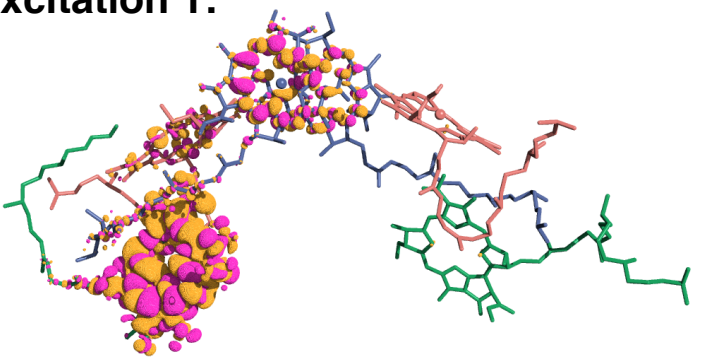

$\lambda=756.89 \mathrm{~nm}$, Localization: $\mathrm{H}_{\mathrm{M}}$ $\int \Delta \rho^{E X_{1}}(\overrightarrow{\mathbf{r}}) d \overrightarrow{\mathbf{r}}=+0.00012 e$

\section{Excitation 2:}

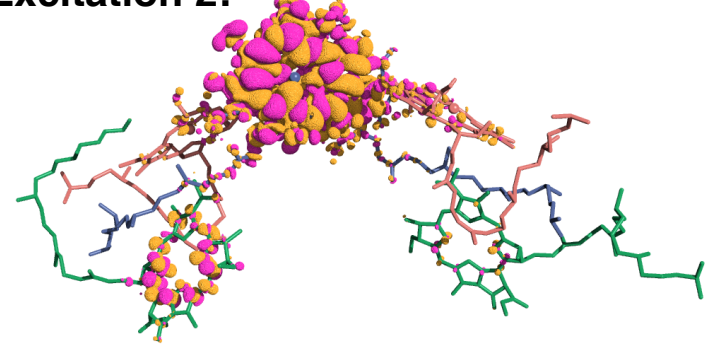

$\lambda=746.81 \mathrm{~nm}$, Localization: $\mathrm{P}$

$\int \Delta \rho^{E X_{2}}(\overrightarrow{\mathbf{r}}) d \overrightarrow{\mathbf{r}}=+0.00087 e$

\section{Excitation 3:}

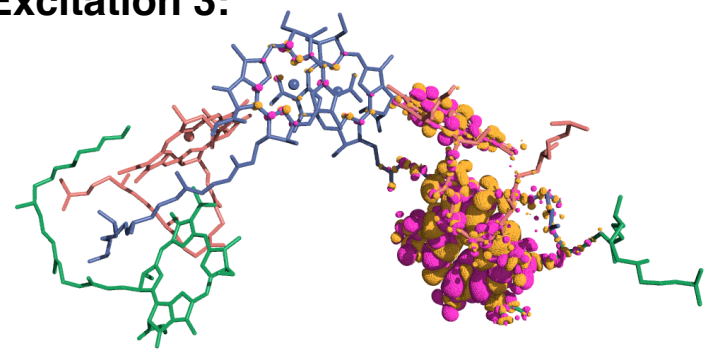

$\lambda=717.63 \mathrm{~nm}$, Localization: $\mathrm{H}_{\mathrm{L}}$ $\int \Delta \rho^{E X_{3}}(\overrightarrow{\mathbf{r}}) d \overrightarrow{\mathbf{r}}=+0.00073 e$

Figure S7. Comparison of the excitation density differences positive isosurface: orange, negative isosurface: pink) from OS-100 TD-DFT calculations with those from FOS TD-DFT calculations for $\mathrm{RC}$ is shown. The first three excitations are shown. All isosurfaces enclose $70 \%$ of the integrated change in $\Delta \rho^{E X}(\vec{r})$. 


\section{Excitation Density Differences}

OS-100

\section{Excitation 4:}

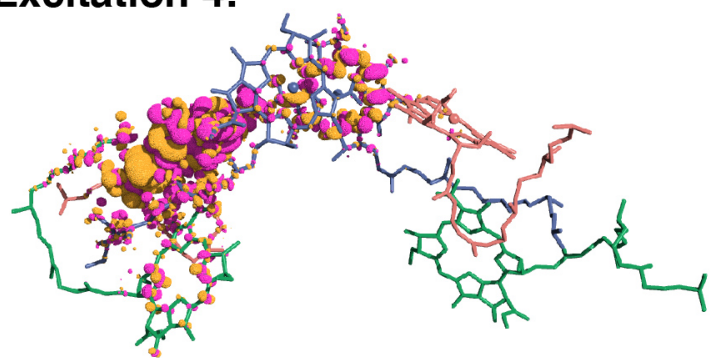

$\lambda=679.86 \mathrm{~nm}$, Localization: $\mathrm{B}_{\mathrm{M}}$

$\int \Delta \rho^{E X_{4}}(\overrightarrow{\mathbf{r}}) d \overrightarrow{\mathbf{r}}=+0.00108 e$

\section{Excitation 5:}

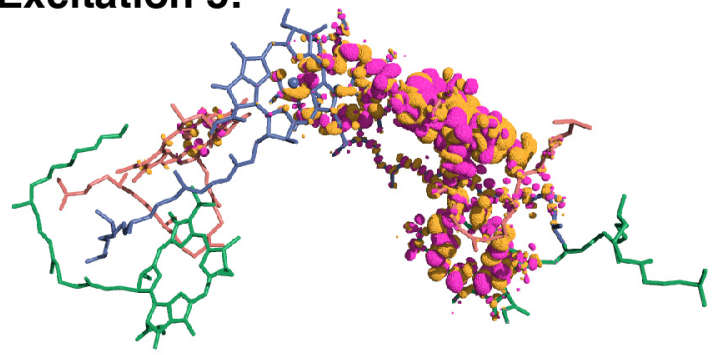

$\lambda=667.71 \mathrm{~nm}$, Localization: $\mathrm{B}$

$\int \Delta \rho^{E X_{5}}(\overrightarrow{\mathbf{r}}) d \overrightarrow{\mathbf{r}}=+0.00060 e$

\section{Excitation 6:}

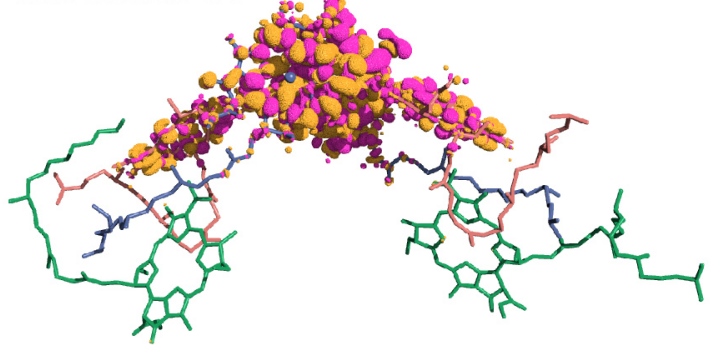

$\lambda=652.11 \mathrm{~nm}$, Localization: $\mathrm{P}$

$\int \Delta \rho^{E X_{6}}(\overrightarrow{\mathbf{r}}) d \overrightarrow{\mathbf{r}}=+0.00103 e$

\section{FOS}

Excitation 4:

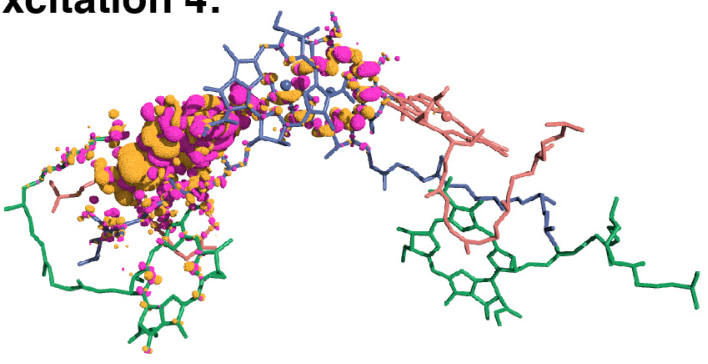

$\lambda=704.51 \mathrm{~nm}$, Localization: $\mathrm{B}_{\mathrm{M}}$ $\int \Delta \rho^{E X_{4}}(\overrightarrow{\mathbf{r}}) d \overrightarrow{\mathbf{r}}=+0.00088 e$

\section{Excitation 5:}

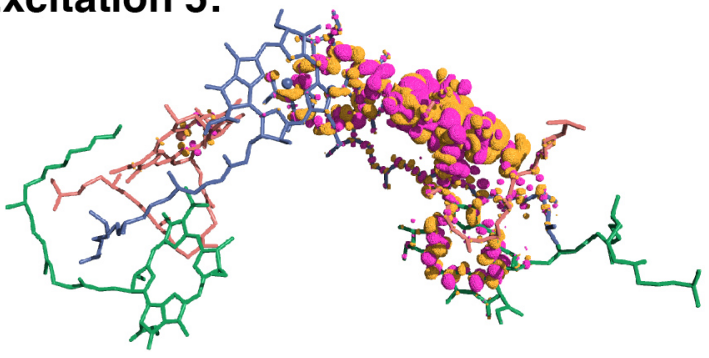

$\lambda=692.20 \mathrm{~nm}$, Localization:

$\int \Delta \rho^{E X_{5}}(\overrightarrow{\mathbf{r}}) d \overrightarrow{\mathbf{r}}=+0.00061 e$

\section{Excitation 6:}

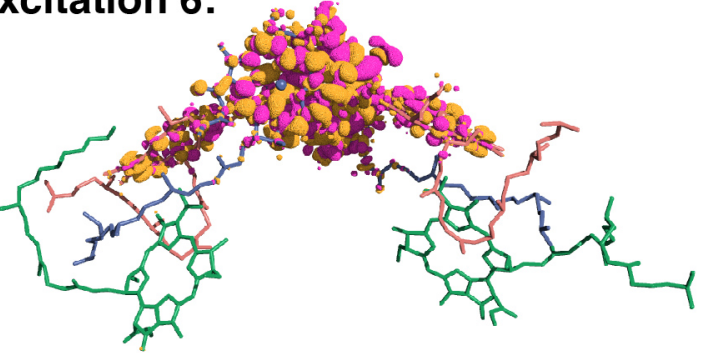

$\lambda=672.91 \mathrm{~nm}$, Localization: $\mathrm{P}$

$\int \Delta \rho^{E X_{6}}(\overrightarrow{\mathbf{r}}) d \overrightarrow{\mathbf{r}}=+0.00090 e$

Figure S8. Comparison of the excitation density differences (positive isosurface: orange, negative isosurface: pink) from OS-100 TD-DFT calculations with those from FOS TD-DFT calculations for RC is shown. The first three excitations are shown. All isosurfaces enclose $70 \%$ of the integrated change in $\Delta \rho^{E X}(\vec{r})$. 
Table S1. Excited state analysis using orbital transition contributions for excitations in the Q band of FbTPP with orbital sizes FOS and OS -100

\begin{tabular}{|c|c|c|c|c|c|c|c|c|c|}
\hline Window Size & $\lambda(\mathrm{nm})$ & $\mathbf{f}$ & Orbital Transition & Orbital Contributions & Window Size & $\lambda(\mathrm{nm})$ & $\mathbf{f}$ & Orbital Transition & Orbital Contributions \\
\hline \multirow[t]{4}{*}{ FOS } & 595.46 & 0.016 & $\mathrm{H} \rightarrow \mathrm{L}+1$ & $60.65 \%$ & \multirow[t]{4}{*}{ OS-100 } & 582.07 & 0.0153 & $\mathrm{H} \rightarrow \mathrm{L}+1$ & $60.47 \%$ \\
\hline & & & $\mathrm{H}-1 \rightarrow \mathrm{L}$ & $39.11 \%$ & & & & $\mathrm{H}-1 \rightarrow \mathrm{L}$ & $38.97 \%$ \\
\hline & 541.86 & 0.0359 & $\mathrm{H} \rightarrow \mathrm{L}$ & $59.51 \%$ & & 520.24 & 0.036 & $\mathrm{H} \rightarrow \mathrm{L}$ & $59.59 \%$ \\
\hline & & & $\mathrm{H}-1 \rightarrow \mathrm{L}+1$ & $40.06 \%$ & & & & $\mathrm{H}-1 \rightarrow \mathrm{L}+1$ & $39.77 \%$ \\
\hline
\end{tabular}

Table S2. Excited state analysis using orbital transition contributions for excitations in the Q band of ZnTPP with orbital sizes FOS and OS -100

\begin{tabular}{|c|c|c|c|c|c|c|c|c|c|}
\hline Window Size & $\lambda(\mathrm{nm})$ & f & Orbital Transition & Orbital Contributions & Window Size & $\lambda(\mathrm{nm})$ & $\mathbf{f}$ & Orbital Transition & Orbital Contributions \\
\hline \multirow[t]{8}{*}{ FOS } & 552.53 & 0.0146 & $\mathrm{H} \rightarrow \mathrm{L}$ & $31.66 \%$ & \multirow[t]{8}{*}{ OS-100 } & 535.74 & 0.0139 & $\mathrm{H} \rightarrow \mathrm{L}$ & $34.19 \%$ \\
\hline & & & $\mathrm{H} \rightarrow \mathrm{L}+1$ & $24.50 \%$ & & & & $\mathrm{H}-1 \rightarrow \mathrm{L}+1$ & $26.13 \%$ \\
\hline & & & $\mathrm{H}-1 \rightarrow \mathrm{L}+1$ & $24.39 \%$ & & & & $\mathrm{H} \rightarrow \mathrm{L}+1$ & $21.99 \%$ \\
\hline & & & $\mathrm{H}-1 \rightarrow \mathrm{L}$ & $18.91 \%$ & & & & $\mathrm{H}-1 \rightarrow \mathrm{L}$ & $16.85 \%$ \\
\hline & 552.46 & 0.0144 & $\mathrm{H} \rightarrow \mathrm{L}+1$ & $31.62 \%$ & & 535.67 & 0.0137 & $\mathrm{H} \rightarrow \mathrm{L}+1$ & $34.14 \%$ \\
\hline & & & $\mathrm{H} \rightarrow \mathrm{L}$ & $24.51 \%$ & & & & $\mathrm{H}-1 \rightarrow \mathrm{L}$ & $26.18 \%$ \\
\hline & & & $\mathrm{H}-1 \rightarrow \mathrm{L}$ & $24.43 \%$ & & & & $\mathrm{H} \rightarrow \mathrm{L}$ & $22.02 \%$ \\
\hline & & & $\mathrm{H}-1 \rightarrow \mathrm{L}+1$ & $18.91 \%$ & & & & $\mathrm{H}-1 \rightarrow \mathrm{L}+1$ & $16.84 \%$ \\
\hline
\end{tabular}

Table S3. Excited state analysis using orbital transition contributions for excitations in the Q band of BC with orbital sizes FOS and OS -100

\begin{tabular}{|c|c|c|c|c|c|c|c|c|c|}
\hline Window Size & $\lambda(\mathrm{nm})$ & $\mathbf{f}$ & Orbital Transition & Orbital Contributions & Window Size & $\lambda(\mathrm{nm})$ & $\mathbf{f}$ & Orbital Transition & Orbital Contributions \\
\hline \multirow[t]{5}{*}{ FOS } & 667.29 & 0.3581 & $\mathrm{H} \rightarrow \mathrm{L}$ & $91.17 \%$ & OS-100 & 645.59 & 0.3588 & $\mathrm{H} \rightarrow \mathrm{L}$ & $76.79 \%$ \\
\hline & & & $\mathrm{H}-1 \rightarrow \mathrm{L}+1$ & $10.09 \%$ & & & & $\mathrm{H}-1 \rightarrow \mathrm{L}+1$ & $10.41 \%$ \\
\hline & & & $\mathrm{H}-1 \leftarrow \mathrm{L}+1$ & $-2.81 \%$ & & & & $\mathrm{H}-1 \leftarrow \mathrm{L}+1$ & $-2.71 \%$ \\
\hline & 497.69 & 0.1027 & $\mathrm{H}-1 \rightarrow \mathrm{L}$ & $75.85 \%$ & & 474.89 & 0.1142 & $\mathrm{H}-1 \rightarrow \mathrm{L}$ & $76.79 \%$ \\
\hline & & & $\mathrm{H} \rightarrow \mathrm{L}+1$ & $24.13 \%$ & & & & $\mathrm{H} \rightarrow \mathrm{L}+1$ & $23.01 \%$ \\
\hline
\end{tabular}


Table S4. Excited state analysis using orbital transition contributions for excitations in the Q band of $\mathrm{BC}-\mathrm{Ph}^{3} \mathrm{Ph}^{13} \mathrm{with}$ orbital sizes FOS and OS -100

\begin{tabular}{|c|c|c|c|c|c|c|c|c|c|}
\hline Window Size & $\lambda(\mathrm{nm})$ & f & Orbital Transition & Orbital Contributions & Window Size & $\lambda(\mathrm{nm})$ & f & Orbital Transition & Orbital Contributions \\
\hline \multirow[t]{5}{*}{ FOS } & 698.07 & 0.5645 & $\mathrm{H} \rightarrow \mathrm{L}$ & $92.05 \%$ & OS-100 & 676.96 & 0.5669 & $\mathrm{H} \rightarrow \mathrm{L}$ & $91.77 \%$ \\
\hline & & & $\mathrm{H}-1 \rightarrow \mathrm{L}+1$ & $8.43 \%$ & & & & $\mathrm{H}-1 \rightarrow \mathrm{L}+1$ & $8.61 \%$ \\
\hline & & & $\mathrm{H}-1 \leftarrow \mathrm{L}+1$ & $-2.62 \%$ & & & & $\mathrm{H}-1 \leftarrow \mathrm{L}+1$ & $-2.54 \%$ \\
\hline & 506.58 & 0.1205 & $\mathrm{H}-1 \rightarrow \mathrm{L}$ & $75.25 \%$ & & 484.2 & 0.1337 & $\mathrm{H}-1 \rightarrow \mathrm{L}$ & $76.09 \%$ \\
\hline & & & $\mathrm{H} \rightarrow \mathrm{L}+1$ & $23.99 \%$ & & & & $\mathrm{H} \rightarrow \mathrm{L}+1$ & $23.01 \%$ \\
\hline
\end{tabular}

Table S5. Excited state analysis using orbital transition contributions for excitations in the Q band of BC-F ${ }^{3} \mathrm{~F}^{13}$ with orbital sizes FOS and OS -100

\begin{tabular}{|c|c|c|c|c|c|c|c|c|c|}
\hline Window Size & $\lambda(\mathrm{nm})$ & f & Orbital Transition & Orbital Contributions & Window Size & $\lambda(\mathrm{nm})$ & f & Orbital Transition & Orbital Contributions \\
\hline \multirow[t]{5}{*}{ FOS } & 729.23 & 0.3964 & $\mathrm{H} \rightarrow \mathrm{L}$ & $93.05 \%$ & OS-100 & 705.38 & 0.4001 & $\mathrm{H} \rightarrow \mathrm{L}$ & $92.80 \%$ \\
\hline & & & $\mathrm{H}-1 \rightarrow \mathrm{L}+1$ & $8.25 \%$ & & & & $\mathrm{H}-1 \rightarrow \mathrm{L}+1$ & $8.46 \%$ \\
\hline & & & $\mathrm{H}-1 \leftarrow \mathrm{L}+1$ & $-2.86 \%$ & & & & $\mathrm{H}-1 \leftarrow \mathrm{L}+1$ & $-2.77 \%$ \\
\hline & 504.9 & 0.0484 & $\mathrm{H}-1 \rightarrow \mathrm{L}$ & $68.88 \%$ & & 483.72 & 0.0524 & $\mathrm{H}-1 \rightarrow \mathrm{L}$ & $69.42 \%$ \\
\hline & & & $\mathrm{H} \rightarrow \mathrm{L}+1$ & $30.95 \%$ & & & & $\mathrm{H} \rightarrow \mathrm{L}+1$ & $30.26 \%$ \\
\hline
\end{tabular}

Table S6. Excited state analysis using orbital transition contributions for excitations in the Q band of Bchl $a$ with orbital sizes FOS and OS -100

\begin{tabular}{|c|c|c|c|c|c|c|c|c|c|}
\hline Window Size & $\lambda(\mathrm{nm})$ & f & Orbital Transition & Orbital Contributions & Window Size & $\lambda(\mathrm{nm})$ & $\mathbf{f}$ & Orbital Transition & Orbital Contributions \\
\hline \multirow[t]{5}{*}{ FOS } & 754.67 & 0.5378 & $\mathrm{H} \rightarrow \mathrm{L}$ & $95.90 \%$ & OS-100 & 724.18 & 0.5362 & $\mathrm{H} \rightarrow \mathrm{L}$ & $96.00 \%$ \\
\hline & & & $\mathrm{H}-1 \rightarrow \mathrm{L}+1$ & $4.42 \%$ & & & & $\mathrm{H}-1 \rightarrow \mathrm{L}+1$ & $4.40 \%$ \\
\hline & & & $\mathrm{H}-1 \leftarrow \mathrm{L}+1$ & $-2.00 \%$ & & & & & \\
\hline & 542.26 & 0.1669 & $\mathrm{H}-1 \rightarrow \mathrm{L}$ & $84.18 \%$ & & 522.25 & 0.172 & $\mathrm{H}-1 \rightarrow \mathrm{L}$ & $84.71 \%$ \\
\hline & & & $\mathrm{H} \rightarrow \mathrm{L}+1$ & $14.44 \%$ & & & & $\mathrm{H} \rightarrow \mathrm{L}+1$ & $13.69 \%$ \\
\hline
\end{tabular}


Table S7. Excited state analysis using orbital transition contributions for excitations in the Q band of FbC-FbB with orbital sizes FOS and OS -100

\begin{tabular}{|c|c|c|c|c|c|c|c|c|c|}
\hline Window Size & $\lambda(\mathbf{n m})$ & f & Orbital Transition & Orbital Contributions & Window Size & $\lambda(\mathbf{n m})$ & f & Orbital Transition & Orbital Contributions \\
\hline \multirow[t]{23}{*}{ FOS } & 714.86 & 0.6099 & $\mathrm{H} \rightarrow \mathrm{L}$ & $56.75 \%$ & OS-100 & 694.02 & 0.612 & $\mathrm{H} \rightarrow \mathrm{L}$ & $56.75 \%$ \\
\hline & & & $\mathrm{H} \rightarrow \mathrm{L}+1$ & $34.94 \%$ & & & & $\mathrm{H} \rightarrow \mathrm{L}+1$ & $34.73 \%$ \\
\hline & & & $\mathrm{H}-2 \rightarrow \mathrm{L}+3$ & $3.34 \%$ & & & & $\mathrm{H}-2 \rightarrow \mathrm{L}+3$ & $3.43 \%$ \\
\hline & & & $\mathrm{H}-1 \rightarrow \mathrm{L}+3$ & $2.99 \%$ & & & & $\mathrm{H}-1 \rightarrow \mathrm{L}+3$ & $3.07 \%$ \\
\hline & 625.14 & 0.4638 & $\mathrm{H}-2 \rightarrow \mathrm{L}+1$ & $23.60 \%$ & & 610.36 & 0.4644 & $\mathrm{H}-2 \rightarrow \mathrm{L}+1$ & $23.43 \%$ \\
\hline & & & $\mathrm{H}-1 \rightarrow \mathrm{L}+1$ & $18.41 \%$ & & & & $\mathrm{H}-2 \rightarrow \mathrm{L}$ & $18.14 \%$ \\
\hline & & & $\mathrm{H}-2 \rightarrow \mathrm{L}$ & $17.82 \%$ & & & & $\mathrm{H}-1 \rightarrow \mathrm{L}+1$ & $18.10 \%$ \\
\hline & & & $\mathrm{H}-1 \rightarrow \mathrm{L}$ & $17.71 \%$ & & & & $\mathrm{H}-1 \rightarrow \mathrm{L}$ & $17.30 \%$ \\
\hline & & & $\mathrm{H}-3 \rightarrow \mathrm{L}+2$ & $13.08 \%$ & & & & $\mathrm{H}-3 \rightarrow \mathrm{L}+2$ & $12.99 \%$ \\
\hline & & & $\mathrm{H}-1 \rightarrow \mathrm{L}+2$ & $3.33 \%$ & & & & $\mathrm{H}-1 \rightarrow \mathrm{L}+1$ & $3.51 \%$ \\
\hline & & & $\mathrm{H}-3 \rightarrow \mathrm{L}+1$ & $2.61 \%$ & & & & $\mathrm{H}-3 \rightarrow \mathrm{L}+1$ & $2.89 \%$ \\
\hline & 561.55 & 0.6182 & $\mathrm{H}-2 \rightarrow \mathrm{L}$ & $28.63 \%$ & & 538.43 & 0.67 & $\mathrm{H}-1 \rightarrow \mathrm{L}$ & $29.35 \%$ \\
\hline & & & $\mathrm{H}-1 \rightarrow \mathrm{L}$ & $27.85 \%$ & & & & $\mathrm{H}-2 \rightarrow \mathrm{L}$ & $29.17 \%$ \\
\hline & & & $\mathrm{H} \rightarrow \mathrm{L}+3$ & $12.20 \%$ & & & & $\mathrm{H}-2 \rightarrow \mathrm{L}+1$ & $11.74 \%$ \\
\hline & & & $\mathrm{H}-2 \rightarrow \mathrm{L}+1$ & $11.96 \%$ & & & & $\mathrm{H}-1 \rightarrow \mathrm{L}+1$ & $11.25 \%$ \\
\hline & & & $\mathrm{H}-1 \rightarrow \mathrm{L}+1$ & $11.78 \%$ & & & & $\mathrm{H} \rightarrow \mathrm{L}+3$ & $11.06 \%$ \\
\hline & & & $\mathrm{H}-3 \rightarrow \mathrm{L}+1$ & $4.60 \%$ & & & & $\mathrm{H}-3 \rightarrow \mathrm{L}+1$ & $4.64 \%$ \\
\hline & 519.85 & 0.14 & $\mathrm{H}-3 \rightarrow \mathrm{L}$ & $26.56 \%$ & & 501.06 & 0.1567 & $\mathrm{H}-3 \rightarrow \mathrm{L}$ & $26.32 \%$ \\
\hline & & & $\mathrm{H}-3 \rightarrow \mathrm{L}+1$ & $25.73 \%$ & & & & $\mathrm{H}-3 \rightarrow \mathrm{L}+1$ & $24.86 \%$ \\
\hline & & & $\mathrm{H}-2 \rightarrow \mathrm{L}+2$ & $15.18 \%$ & & & & $\mathrm{H}-2 \rightarrow \mathrm{L}+2$ & $14.99 \%$ \\
\hline & & & $\mathrm{H}-1 \rightarrow \mathrm{L}+1$ & $13.13 \%$ & & & & $\mathrm{H}-1 \rightarrow \mathrm{L}+1$ & $14.71 \%$ \\
\hline & & & $\mathrm{H}-1 \rightarrow \mathrm{L}+2$ & $12.18 \%$ & & & & $\mathrm{H}-1 \rightarrow \mathrm{L}+2$ & $12.01 \%$ \\
\hline & & & $\mathrm{H}-1 \rightarrow \mathrm{L}$ & $2.07 \%$ & & & & $\mathrm{H}-1 \rightarrow \mathrm{L}$ & $2.14 \%$ \\
\hline
\end{tabular}


Table S8. Excited state analysis using orbital transition contributions for excitations in the Q band of ZnFb with orbital sizes FOS and OS-100

\begin{tabular}{|c|c|c|c|c|c|c|c|c|c|}
\hline Window Size & $\lambda(\mathrm{nm})$ & f & Orbital Transition & Orbital Contributions & Window Size & $\lambda(\mathrm{nm})$ & f & Orbital Transition & Orbital Contributions \\
\hline \multirow[t]{12}{*}{ FOS } & 594.41 & 0.0247 & $\mathrm{H}-1 \rightarrow \mathrm{L}$ & $38.07 \%$ & OS-100 & 580.01 & 0.023 & $\mathrm{H}-1 \rightarrow \mathrm{L}$ & $37.88 \%$ \\
\hline & & & $\mathrm{H}-3 \rightarrow \mathrm{L}+1$ & $24.93 \%$ & & & & $\mathrm{H}-3 \rightarrow \mathrm{L}+1$ & $24.88 \%$ \\
\hline & & & $\mathrm{H}-1 \rightarrow \mathrm{L}+1$ & $19.67 \%$ & & & & $\mathrm{H}-1 \rightarrow \mathrm{L}+1$ & $19.58 \%$ \\
\hline & & & $\mathrm{H}-3 \rightarrow \mathrm{L}$ & $13.66 \%$ & & & & $\mathrm{H}-3 \rightarrow \mathrm{L}$ & $13.67 \%$ \\
\hline & 554.89 & 0.0416 & $\mathrm{H} \rightarrow \mathrm{L}+2$ & $54.31 \%$ & & 536.9 & 0.0385 & $\mathrm{H} \rightarrow \mathrm{L}+2$ & $54.25 \%$ \\
\hline & & & $\mathrm{H}-2 \rightarrow \mathrm{L}+3$ & $41.61 \%$ & & & & $\mathrm{H}-2 \rightarrow \mathrm{L}+3$ & $41.48 \%$ \\
\hline & & & $\mathrm{H}-1 \rightarrow \mathrm{L}+2$ & $2.05 \%$ & & & & $\mathrm{H}-1 \rightarrow \mathrm{L}+2$ & $2.01 \%$ \\
\hline & & & $\mathrm{H}-2 \rightarrow \mathrm{L}+2$ & $43.39 \%$ & & & & $\mathrm{H}-2 \rightarrow \mathrm{L}+2$ & $43.44 \%$ \\
\hline & 540.88 & 0.0389 & $\mathrm{H}-1 \rightarrow \mathrm{L}+1$ & $35.44 \%$ & & 517.78 & 0.0393 & $\mathrm{H}-1 \rightarrow \mathrm{L}+1$ & $35.26 \%$ \\
\hline & & & $\mathrm{H}-3 \rightarrow \mathrm{L}$ & $27.51 \%$ & & & & $\mathrm{H}-3 \rightarrow \mathrm{L}$ & $27.57 \%$ \\
\hline & & & $\mathrm{H}-1 \rightarrow \mathrm{L}$ & $19.27 \%$ & & & & $\mathrm{H}-1 \rightarrow \mathrm{L}$ & $19.32 \%$ \\
\hline & & & $\mathrm{H}-3 \rightarrow \mathrm{L}+1$ & $13.88 \%$ & & & & $\mathrm{H}-3 \rightarrow \mathrm{L}+1$ & $13.77 \%$ \\
\hline
\end{tabular}


Table S9. Excited state analysis using orbital transition contributions for excitations in the Q band of ZnZnFb with orbital sizes FOS and OS -100

\begin{tabular}{|c|c|c|c|c|c|c|c|c|c|}
\hline $\begin{array}{c}\text { Window } \\
\text { Size }\end{array}$ & $\lambda(\mathbf{n m})$ & $\mathbf{f}$ & Orbital Transition & Orbital Contributions & Window Size & $\lambda(\mathbf{n m})$ & $\mathbf{f}$ & Orbital Transition & Orbital Contributions \\
\hline \multirow[t]{26}{*}{ FOS } & 594.29 & 0.025 & $\mathrm{H}-2 \rightarrow \mathrm{L}$ & $35.52 \%$ & OS-100 & 580.02 & 0.0232 & $\mathrm{H}-2 \rightarrow \mathrm{L}$ & $35.32 \%$ \\
\hline & & & $\mathrm{H}-5 \rightarrow \mathrm{L}+1$ & $23.39 \%$ & & & & $\mathrm{H}-5 \rightarrow \mathrm{L}+1$ & $23.31 \%$ \\
\hline & & & $\mathrm{H}-2 \rightarrow \mathrm{L}+1$ & $21.81 \%$ & & & & $\mathrm{H}-2 \rightarrow \mathrm{L}+1$ & $21.76 \%$ \\
\hline & & & $\mathrm{H}-5 \rightarrow \mathrm{L}$ & $14.98 \%$ & & & & $\mathrm{H}-5 \rightarrow \mathrm{L}$ & $15.00 \%$ \\
\hline & 557.46 & 0.1128 & $\mathrm{H}-4 \rightarrow \mathrm{L}+4$ & $37.64 \%$ & & 539.8 & 0.1072 & $\mathrm{H}-4 \rightarrow \mathrm{L}+4$ & $37.84 \%$ \\
\hline & & & $\mathrm{H} \rightarrow \mathrm{L}+2$ & $27.52 \%$ & & & & $\mathrm{H} \rightarrow \mathrm{L}+2$ & $27.39 \%$ \\
\hline & & & $\mathrm{H}-1 \rightarrow \mathrm{L}+2$ & $20.25 \%$ & & & & $\mathrm{H}-1 \rightarrow \mathrm{L}+2$ & $21.06 \%$ \\
\hline & & & $\mathrm{H}-1 \rightarrow \mathrm{L}+3$ & $7.54 \%$ & & & & $\mathrm{H}-1 \rightarrow \mathrm{L}+3$ & $6.77 \%$ \\
\hline & 554.12 & 0.0142 & $\mathrm{H}-3 \rightarrow \mathrm{L}+5$ & $40.23 \%$ & & 536.21 & 0.0155 & $\mathrm{H}-3 \rightarrow \mathrm{L}+5$ & $40.58 \%$ \\
\hline & & & $\mathrm{H} \rightarrow \mathrm{L}+3$ & $29.24 \%$ & & & & $\mathrm{H} \rightarrow \mathrm{L}+3$ & $29.01 \%$ \\
\hline & & & $\mathrm{H}-1 \rightarrow \mathrm{L}+3$ & $19.34 \%$ & & & & $\mathrm{H}-1 \rightarrow \mathrm{L}+3$ & $19.99 \%$ \\
\hline & & & $\mathrm{H}-1 \rightarrow \mathrm{L}+2$ & $7.43 \%$ & & & & $\mathrm{H}-1 \rightarrow \mathrm{L}+2$ & $6.67 \%$ \\
\hline & & & $\mathrm{H}-4 \rightarrow \mathrm{L}+4$ & $2.11 \%$ & & & & & \\
\hline & 552.67 & 0.0065 & $\mathrm{H}-4 \rightarrow \mathrm{L}+2$ & $38.56 \%$ & & 534.18 & 0.0053 & $\mathrm{H}-4 \rightarrow \mathrm{L}+2$ & $38.57 \%$ \\
\hline & & & $\mathrm{H}-1 \rightarrow \mathrm{L}+4$ & $26.73 \%$ & & & & $\mathrm{H}-1 \rightarrow \mathrm{L}+4$ & $26.55 \%$ \\
\hline & & & $\mathrm{H} \rightarrow \mathrm{L}+4$ & $25.74 \%$ & & & & $\mathrm{H} \rightarrow \mathrm{L}+4$ & $25.66 \%$ \\
\hline & & & $\mathrm{H}-4 \rightarrow \mathrm{L}+3$ & $4.40 \%$ & & & & $\mathrm{H}-4 \rightarrow \mathrm{L}+3$ & $4.38 \%$ \\
\hline & & & $\mathrm{H}-2 \rightarrow \mathrm{L}+4$ & $2.06 \%$ & & & & $\mathrm{H}-2 \rightarrow \mathrm{L}+4$ & $2.03 \%$ \\
\hline & 552.18 & 0.0077 & $\mathrm{H}-3 \rightarrow \mathrm{L}+3$ & $39.35 \%$ & & 533.87 & 0.0063 & $\mathrm{H}-3 \rightarrow \mathrm{L}+3$ & $39.84 \%$ \\
\hline & & & $\mathrm{H} \rightarrow \mathrm{L}+5$ & $29.03 \%$ & & & & $\mathrm{H} \rightarrow \mathrm{L}+5$ & $28.88 \%$ \\
\hline & & & $\mathrm{H}-1 \rightarrow \mathrm{L}+5$ & $25.97 \%$ & & & & $\mathrm{H}-1 \rightarrow \mathrm{L}+5$ & $25.76 \%$ \\
\hline & & & $\mathrm{H}-3 \rightarrow \mathrm{L}+2$ & $4.66 \%$ & & & & $\mathrm{H}-3 \rightarrow \mathrm{L}+2$ & $4.68 \%$ \\
\hline & 540.9 & 0.0402 & $\mathrm{H}-2 \rightarrow \mathrm{L}+1$ & $33.23 \%$ & & 517.96 & 0.041 & $\mathrm{H}-2 \rightarrow \mathrm{L}+1$ & $33.04 \%$ \\
\hline & & & $\mathrm{H}-6 \rightarrow \mathrm{L}$ & $25.71 \%$ & & & & H-6 $\rightarrow$ L & $25.73 \%$ \\
\hline & & & $\mathrm{H}-2 \rightarrow \mathrm{L}$ & $21.10 \%$ & & & & $\mathrm{H}-2 \rightarrow \mathrm{L}$ & $21.18 \%$ \\
\hline & & & $\mathrm{H}-6 \rightarrow \mathrm{L}+1$ & $15.44 \%$ & & & & $\mathrm{H}-6 \rightarrow \mathrm{L}+1$ & $15.34 \%$ \\
\hline
\end{tabular}


Table S10. Excited state analysis using orbital transition contributions for excitations in the Q band of FbTPP with orbital sizes FOS and OS-60

\begin{tabular}{|c|c|c|c|c|c|c|c|c|c|}
\hline Window Size & $\lambda(\mathrm{nm})$ & $\mathbf{f}$ & Orbital Transition & Orbital Contributions & Window Size & $\lambda(\mathrm{nm})$ & $\mathbf{f}$ & Orbital Transition & Orbital Contributions \\
\hline \multirow[t]{4}{*}{ FOS } & 595.46 & 0.016 & $\mathrm{H} \rightarrow \mathrm{L}+1$ & $60.65 \%$ & \multirow[t]{4}{*}{ OS-60 } & 565.64 & 0.0118 & $\mathrm{H} \rightarrow \mathrm{L}+1$ & $59.06 \%$ \\
\hline & & & $\mathrm{H}-1 \rightarrow \mathrm{L}$ & $39.11 \%$ & & & & $\mathrm{H}-1 \rightarrow \mathrm{L}$ & $39.99 \%$ \\
\hline & 541.86 & 0.0359 & $\mathrm{H} \rightarrow \mathrm{L}$ & $59.51 \%$ & & 495.73 & 0.0265 & $\mathrm{H} \rightarrow \mathrm{L}$ & $58.29 \%$ \\
\hline & & & $\mathrm{H}-1 \rightarrow \mathrm{L}+1$ & $40.06 \%$ & & & & $\mathrm{H}-1 \rightarrow \mathrm{L}+1$ & $40.88 \%$ \\
\hline
\end{tabular}

Table S11. Excited state analysis using orbital transition contributions for excitations in the Q band of ZnTPP with orbital sizes FOS and OS-60

\begin{tabular}{|c|c|c|c|c|c|c|c|c|c|}
\hline Window Size & $\lambda(\mathrm{nm})$ & $\mathbf{f}$ & Orbital Transition & Orbital Contributions & Window Size & $\lambda(\mathrm{nm})$ & $\mathbf{f}$ & Orbital Transition & Orbital Contributions \\
\hline \multirow[t]{8}{*}{ FOS } & 552.53 & 0.0146 & $\mathrm{H} \rightarrow \mathrm{L}$ & $31.66 \%$ & \multirow[t]{8}{*}{ OS-60 } & \multirow[t]{4}{*}{516.24} & \multirow[t]{4}{*}{0.0093} & $\mathrm{H} \rightarrow \mathrm{L}+1$ & $53.58 \%$ \\
\hline & & & $\mathrm{H} \rightarrow \mathrm{L}+1$ & $24.50 \%$ & & & & \multirow[t]{3}{*}{$\mathrm{H}-1 \rightarrow \mathrm{L}$} & \multirow[t]{3}{*}{$43.21 \%$} \\
\hline & & & $\mathrm{H}-1 \rightarrow \mathrm{L}+1$ & $24.39 \%$ & & & & & \\
\hline & & & $\mathrm{H}-1 \rightarrow \mathrm{L}$ & $18.91 \%$ & & & & & \\
\hline & 552.46 & 0.0144 & $\mathrm{H} \rightarrow \mathrm{L}+1$ & $31.62 \%$ & & \multirow[t]{4}{*}{516.21} & \multirow[t]{4}{*}{0.0096} & $\mathrm{H} \rightarrow \mathrm{L}$ & $53.60 \%$ \\
\hline & & & $\mathrm{H} \rightarrow \mathrm{L}$ & $24.51 \%$ & & & & $\mathrm{H}-1 \rightarrow \mathrm{L}+1$ & $43.19 \%$ \\
\hline & & & $\mathrm{H}-1 \rightarrow \mathrm{L}$ & $24.43 \%$ & & & & & \\
\hline & & & $\mathrm{H}-1 \rightarrow \mathrm{L}+1$ & $18.91 \%$ & & & & & \\
\hline
\end{tabular}

Table S12. Excited state analysis using orbital transition contributions for excitations in the Q band of BC with orbital sizes FOS and OS-60

\begin{tabular}{|c|c|c|c|c|c|c|c|c|c|}
\hline Window Size & $\lambda(\mathrm{nm})$ & $\mathbf{f}$ & Orbital Transition & Orbital Contributions & Window Size & $\lambda(\mathrm{nm})$ & $\mathbf{f}$ & Orbital Transition & Orbital Contributions \\
\hline \multirow[t]{5}{*}{ FOS } & 667.29 & 0.3581 & $\mathrm{H} \rightarrow \mathrm{L}$ & $91.17 \%$ & OS-60 & 616.12 & 0.3575 & $\mathrm{H} \rightarrow \mathrm{L}$ & $89.19 \%$ \\
\hline & & & $\mathrm{H}-1 \rightarrow \mathrm{L}+1$ & $10.09 \%$ & & & & $\mathrm{H}-1 \rightarrow \mathrm{L}+1$ & $12.09 \%$ \\
\hline & & & $\mathrm{H}-1 \leftarrow \mathrm{L}+1$ & $-2.81 \%$ & & & & $\mathrm{H}-1 \leftarrow \mathrm{L}+1$ & $-2.73 \%$ \\
\hline & 497.69 & 0.1027 & $\mathrm{H}-1 \rightarrow \mathrm{L}$ & $75.85 \%$ & & 452.75 & 0.1113 & $\mathrm{H}-1 \rightarrow \mathrm{L}$ & $75.56 \%$ \\
\hline & & & $\mathrm{H} \rightarrow \mathrm{L}+1$ & $24.13 \%$ & & & & $\mathrm{H} \rightarrow \mathrm{L}+1$ & $24.17 \%$ \\
\hline
\end{tabular}


Table S13. Excited state analysis using orbital transition contributions for excitations in the Q band of $\mathrm{BC}-\mathrm{Ph}^{3} \mathrm{Ph}^{13} \mathrm{with}$ orbital sizes FOS and OS-60

\begin{tabular}{|c|c|c|c|c|c|c|c|c|c|}
\hline Window Size & $\lambda(\mathrm{nm})$ & f & Orbital Transition & Orbital Contributions & Window Size & $\lambda(\mathrm{nm})$ & f & Orbital Transition & Orbital Contributions \\
\hline \multirow[t]{5}{*}{ FOS } & 698.07 & 0.5645 & $\mathrm{H} \rightarrow \mathrm{L}$ & $92.05 \%$ & OS-60 & 644.79 & 0.5510 & $\mathrm{H} \rightarrow \mathrm{L}$ & $90.43 \%$ \\
\hline & & & $\mathrm{H}-1 \rightarrow \mathrm{L}+1$ & $8.43 \%$ & & & & $\mathrm{H}-1 \rightarrow \mathrm{L}+1$ & $9.97 \%$ \\
\hline & & & $\mathrm{H}-1 \leftarrow \mathrm{L}+1$ & $-2.62 \%$ & & & & $\mathrm{H}-1 \leftarrow \mathrm{L}+1$ & $-2.55 \%$ \\
\hline & 506.58 & 0.1205 & $\mathrm{H}-1 \rightarrow \mathrm{L}$ & $75.25 \%$ & & 462.12 & 0.1385 & $\mathrm{H}-1 \rightarrow \mathrm{L}$ & $75.11 \%$ \\
\hline & & & $\mathrm{H} \rightarrow \mathrm{L}+1$ & $23.99 \%$ & & & & $\mathrm{H} \rightarrow \mathrm{L}+1$ & $23.92 \%$ \\
\hline
\end{tabular}

Table S14. Excited state analysis using orbital transition contributions for excitations in the Q band of $\mathrm{BC}-\mathrm{F}^{3} \mathrm{~F}^{13}$ with orbital sizes FOS and OS-60

\begin{tabular}{|c|c|c|c|c|c|c|c|c|c|}
\hline Window Size & $\lambda(\mathrm{nm})$ & f & Orbital Transition & Orbital Contributions & Window Size & $\lambda(\mathrm{nm})$ & f & Orbital Transition & Orbital Contributions \\
\hline \multirow[t]{5}{*}{ FOS } & 729.23 & 0.3964 & $\mathrm{H} \rightarrow \mathrm{L}$ & $93.05 \%$ & \multirow[t]{5}{*}{ OS-60 } & \multirow[t]{3}{*}{673.19} & \multirow[t]{3}{*}{0.3997} & $\mathrm{H} \rightarrow \mathrm{L}$ & $91.81 \%$ \\
\hline & & & $\mathrm{H}-1 \rightarrow \mathrm{L}+1$ & $8.25 \%$ & & & & $\mathrm{H}-1 \rightarrow \mathrm{L}+1$ & $9.56 \%$ \\
\hline & & & $\mathrm{H}-1 \leftarrow \mathrm{L}+1$ & $-2.86 \%$ & & & & $\mathrm{H}-1 \leftarrow \mathrm{L}+1$ & $-2.79 \%$ \\
\hline & 504.9 & 0.0484 & $\mathrm{H}-1 \rightarrow \mathrm{L}$ & $68.88 \%$ & & \multirow[t]{2}{*}{460.85} & \multirow[t]{2}{*}{0.0537} & $\mathrm{H}-1 \rightarrow \mathrm{L}$ & $68.73 \%$ \\
\hline & & & $\mathrm{H} \rightarrow \mathrm{L}+1$ & $30.95 \%$ & & & & $\mathrm{H} \rightarrow \mathrm{L}+1$ & $30.84 \%$ \\
\hline
\end{tabular}

Table S15. Excited state analysis using orbital transition contributions for excitations in the Q band of Bchl $a$ with orbital sizes FOS and OS-60

\begin{tabular}{|c|c|c|c|c|c|c|c|c|c|}
\hline Window Size & $\lambda(\mathbf{n m})$ & $\mathbf{f}$ & Orbital Transition & Orbital Contributions & Window Size & $\lambda(\mathrm{nm})$ & f & Orbital Transition & Orbital Contributions \\
\hline \multirow[t]{5}{*}{ FOS } & 754.67 & 0.5378 & $\mathrm{H} \rightarrow \mathrm{L}$ & $95.90 \%$ & OS-60 & 676.83 & 0.5575 & $\mathrm{H} \rightarrow \mathrm{L}$ & $95.59 \%$ \\
\hline & & & $\mathrm{H}-1 \rightarrow \mathrm{L}+1$ & $4.42 \%$ & & & & $\mathrm{H}-1 \rightarrow \mathrm{L}+1$ & $5.12 \%$ \\
\hline & & & $\mathrm{H}-1 \leftarrow \mathrm{L}+1$ & $-2.00 \%$ & & & & & \\
\hline & 542.26 & 0.1669 & $\mathrm{H}-1 \rightarrow \mathrm{L}$ & $84.18 \%$ & & 494.90 & 0.1555 & $\mathrm{H}-1 \rightarrow \mathrm{L}$ & $82.11 \%$ \\
\hline & & & $\mathrm{H} \rightarrow \mathrm{L}+1$ & $14.44 \%$ & & & & $\mathrm{H} \rightarrow \mathrm{L}+1$ & $15.90 \%$ \\
\hline
\end{tabular}


Table S16. Excited state analysis using orbital transition contributions for excitations in the Q band of FbC-FbB with orbital sizes FOS and OS-60

\begin{tabular}{|c|c|c|c|c|c|c|c|c|c|}
\hline Window Size & $\lambda(\mathrm{nm})$ & f & Orbital Transition & Orbital Contributions & Window Size & $\lambda(\mathrm{nm})$ & $\mathbf{f}$ & Orbital Transition & Orbital Contributions \\
\hline \multirow[t]{23}{*}{ FOS } & 714.86 & 0.6099 & $\mathrm{H} \rightarrow \mathrm{L}$ & $56.75 \%$ & OS-60 & 661.13 & 0.5816 & $\mathrm{H} \rightarrow \mathrm{L}$ & $56.21 \%$ \\
\hline & & & $\mathrm{H} \rightarrow \mathrm{L}+1$ & $34.94 \%$ & & & & $\mathrm{H} \rightarrow \mathrm{L}+1$ & $33.97 \%$ \\
\hline & & & $\mathrm{H}-2 \rightarrow \mathrm{L}+3$ & $3.34 \%$ & & & & $\mathrm{H}-2 \rightarrow \mathrm{L}+3$ & $4.04 \%$ \\
\hline & & & $\mathrm{H}-1 \rightarrow \mathrm{L}+3$ & $2.99 \%$ & & & & $\mathrm{H}-1 \rightarrow \mathrm{L}+3$ & $3.62 \%$ \\
\hline & 625.14 & 0.4638 & $\mathrm{H}-2 \rightarrow \mathrm{L}+1$ & $23.60 \%$ & & 592.07 & 0.4488 & $\mathrm{H}-2 \rightarrow \mathrm{L}+1$ & $23.04 \%$ \\
\hline & & & $\mathrm{H}-1 \rightarrow \mathrm{L}+1$ & $18.41 \%$ & & & & $\mathrm{H}-2 \rightarrow \mathrm{L}$ & $18.19 \%$ \\
\hline & & & $\mathrm{H}-2 \rightarrow \mathrm{L}$ & $17.82 \%$ & & & & $\mathrm{H}-1 \rightarrow \mathrm{L}+1$ & $17.16 \%$ \\
\hline & & & $\mathrm{H}-1 \rightarrow \mathrm{L}$ & $17.71 \%$ & & & & $\mathrm{H}-1 \rightarrow \mathrm{L}$ & $16.66 \%$ \\
\hline & & & $\mathrm{H}-3 \rightarrow \mathrm{L}+2$ & $13.08 \%$ & & & & $\mathrm{H}-3 \rightarrow \mathrm{L}+2$ & $13.56 \%$ \\
\hline & & & $\mathrm{H}-1 \rightarrow \mathrm{L}+2$ & $3.33 \%$ & & & & $\mathrm{H}-1 \rightarrow \mathrm{L}+1$ & $4.04 \%$ \\
\hline & & & $\mathrm{H}-3 \rightarrow \mathrm{L}+1$ & $2.61 \%$ & & & & $\mathrm{H}-3 \rightarrow \mathrm{L}+1$ & $3.34 \%$ \\
\hline & 561.55 & 0.6182 & $\mathrm{H}-2 \rightarrow \mathrm{L}$ & $28.63 \%$ & & 513.92 & 0.6870 & $\mathrm{H}-1 \rightarrow \mathrm{L}$ & $30.78 \%$ \\
\hline & & & $\mathrm{H}-1 \rightarrow \mathrm{L}$ & $27.85 \%$ & & & & $\mathrm{H}-2 \rightarrow \mathrm{L}$ & $29.47 \%$ \\
\hline & & & $\mathrm{H} \rightarrow \mathrm{L}+3$ & $12.20 \%$ & & & & $\mathrm{H}-2 \rightarrow \mathrm{L}+1$ & $11.11 \%$ \\
\hline & & & $\mathrm{H}-2 \rightarrow \mathrm{L}+1$ & $11.96 \%$ & & & & $\mathrm{H}-1 \rightarrow \mathrm{L}+1$ & $11.09 \%$ \\
\hline & & & $\mathrm{H}-1 \rightarrow \mathrm{L}+1$ & $11.78 \%$ & & & & $\mathrm{H} \rightarrow \mathrm{L}+3$ & $10.31 \%$ \\
\hline & & & $\mathrm{H}-3 \rightarrow \mathrm{L}+1$ & $4.60 \%$ & & & & $\mathrm{H}-3 \rightarrow \mathrm{L}+1$ & $4.56 \%$ \\
\hline & 519.85 & 0.14 & $\mathrm{H}-3 \rightarrow \mathrm{L}$ & $26.56 \%$ & & 481.06 & 0.1837 & $\mathrm{H}-3 \rightarrow \mathrm{L}$ & $25.43 \%$ \\
\hline & & & $\mathrm{H}-3 \rightarrow \mathrm{L}+1$ & $25.73 \%$ & & & & $\mathrm{H}-3 \rightarrow \mathrm{L}+1$ & $23.14 \%$ \\
\hline & & & $\mathrm{H}-2 \rightarrow \mathrm{L}+2$ & $15.18 \%$ & & & & $\mathrm{H}-2 \rightarrow \mathrm{L}+2$ & $15.11 \%$ \\
\hline & & & $\mathrm{H}-1 \rightarrow \mathrm{L}+1$ & $13.13 \%$ & & & & $\mathrm{H}-1 \rightarrow \mathrm{L}+1$ & $17.07 \%$ \\
\hline & & & $\mathrm{H}-1 \rightarrow \mathrm{L}+2$ & $12.18 \%$ & & & & $\mathrm{H}-1 \rightarrow \mathrm{L}+2$ & $12.13 \%$ \\
\hline & & & $\mathrm{H}-1 \rightarrow \mathrm{L}$ & $2.07 \%$ & & & & $\mathrm{H}-1 \rightarrow \mathrm{L}$ & $2.29 \%$ \\
\hline
\end{tabular}


Table S17. Excited state analysis using orbital transition contributions for excitations in the Q band of ZnFb with orbital sizes FOS and OS-60

\begin{tabular}{|c|c|c|c|c|c|c|c|c|c|}
\hline Window Size & $\lambda(\mathbf{n m})$ & $\mathbf{f}$ & Orbital Transition & Orbital Contributions & Window Size & $\lambda(\mathbf{n m})$ & $\mathbf{f}$ & Orbital Transition & Orbital Contributions \\
\hline \multirow[t]{13}{*}{ FOS } & 594.41 & 0.0247 & $\mathrm{H}-1 \rightarrow \mathrm{L}$ & $38.07 \%$ & OS-60 & 562.85 & 0.0157 & $\mathrm{H}-1 \rightarrow \mathrm{L}$ & $36.41 \%$ \\
\hline & & & $\mathrm{H}-3 \rightarrow \mathrm{L}+1$ & $24.93 \%$ & & & & $\mathrm{H}-3 \rightarrow \mathrm{L}+1$ & $25.62 \%$ \\
\hline & & & $\mathrm{H}-1 \rightarrow \mathrm{L}+1$ & $19.67 \%$ & & & & $\mathrm{H}-1 \rightarrow \mathrm{L}+1$ & $19.30 \%$ \\
\hline & & & $\mathrm{H}-3 \rightarrow \mathrm{L}$ & $13.66 \%$ & & & & $\mathrm{H}-3 \rightarrow \mathrm{L}$ & $14.36 \%$ \\
\hline & 554.89 & 0.0416 & $\mathrm{H} \rightarrow \mathrm{L}+2$ & $54.31 \%$ & & 516.21 & 0.0264 & $\mathrm{H} \rightarrow \mathrm{L}+2$ & $52.61 \%$ \\
\hline & & & $\mathrm{H}-2 \rightarrow \mathrm{L}+3$ & $41.61 \%$ & & & & $\mathrm{H}-2 \rightarrow \mathrm{L}+3$ & $42.91 \%$ \\
\hline & & & $\mathrm{H}-1 \rightarrow \mathrm{L}+2$ & $2.05 \%$ & & & & & \\
\hline & 552.55 & 0.0074 & $\mathrm{H} \rightarrow \mathrm{L}+3$ & $53.00 \%$ & & 514.01 & 0.0025 & $\mathrm{H} \rightarrow \mathrm{L}+3$ & $51.05 \%$ \\
\hline & & & $\mathrm{H}-2 \rightarrow \mathrm{L}+2$ & $43.39 \%$ & & & & $\mathrm{H}-2 \rightarrow \mathrm{L}+2$ & $44.73 \%$ \\
\hline & 540.88 & 0.0389 & $\mathrm{H}-1 \rightarrow \mathrm{L}+1$ & $35.44 \%$ & & 492.48 & 0.0291 & $\mathrm{H}-1 \rightarrow \mathrm{L}+1$ & $34.00 \%$ \\
\hline & & & $\mathrm{H}-3 \rightarrow \mathrm{L}$ & $27.51 \%$ & & & & $\mathrm{H}-3 \rightarrow \mathrm{L}$ & $28.21 \%$ \\
\hline & & & $\mathrm{H}-1 \rightarrow \mathrm{L}$ & $19.27 \%$ & & & & $\mathrm{H}-1 \rightarrow \mathrm{L}$ & $19.18 \%$ \\
\hline & & & $\mathrm{H}-3 \rightarrow \mathrm{L}+1$ & $13.88 \%$ & & & & $\mathrm{H}-3 \rightarrow \mathrm{L}+1$ & $14.40 \%$ \\
\hline
\end{tabular}


Table S18. Excited state analysis using orbital transition contributions for excitations in the Q band of ZnZnFb with orbital sizes FOS and OS -60

\begin{tabular}{|c|c|c|c|c|c|c|c|c|c|}
\hline $\begin{array}{c}\text { Window } \\
\text { Size }\end{array}$ & $\lambda(\mathrm{nm})$ & f & Orbital Transition & Orbital Contributions & Window Size & $\lambda(\mathbf{n m})$ & f & Orbital Transition & Orbital Contributions \\
\hline \multirow[t]{26}{*}{ FOS } & 594.29 & 0.025 & $\mathrm{H}-2 \rightarrow \mathrm{L}$ & $35.52 \%$ & OS-60 & 580.02 & 0.0156 & $\mathrm{H}-2 \rightarrow \mathrm{L}$ & $33.93 \%$ \\
\hline & & & $\mathrm{H}-5 \rightarrow \mathrm{L}+1$ & $23.39 \%$ & & & & $\mathrm{H}-5 \rightarrow \mathrm{L}+1$ & $23.99 \%$ \\
\hline & & & $\mathrm{H}-2 \rightarrow \mathrm{L}+1$ & $21.81 \%$ & & & & $\mathrm{H}-2 \rightarrow \mathrm{L}+1$ & $21.44 \%$ \\
\hline & & & $\mathrm{H}-5 \rightarrow \mathrm{L}$ & $14.98 \%$ & & & & $\mathrm{H}-5 \rightarrow \mathrm{L}$ & $15.72 \%$ \\
\hline & 557.46 & 0.1128 & $\mathrm{H}-4 \rightarrow \mathrm{L}+4$ & $37.64 \%$ & & 519.12 & 0.0780 & $\mathrm{H}-4 \rightarrow \mathrm{L}+4$ & $39.61 \%$ \\
\hline & & & $\mathrm{H} \rightarrow \mathrm{L}+2$ & $27.52 \%$ & & & & $\mathrm{H} \rightarrow \mathrm{L}+2$ & $26.45 \%$ \\
\hline & & & $\mathrm{H}-1 \rightarrow \mathrm{L}+2$ & $20.25 \%$ & & & & $\mathrm{H}-1 \rightarrow \mathrm{L}+2$ & $20.91 \%$ \\
\hline & & & $\mathrm{H}-1 \rightarrow \mathrm{L}+3$ & $7.54 \%$ & & & & $\mathrm{H}-1 \rightarrow \mathrm{L}+3$ & $6.03 \%$ \\
\hline & 554.12 & 0.0142 & $\mathrm{H}-3 \rightarrow \mathrm{L}+5$ & $40.23 \%$ & & 515.87 & 0.0104 & $\mathrm{H}-3 \rightarrow \mathrm{L}+5$ & $42.40 \%$ \\
\hline & & & $\mathrm{H} \rightarrow \mathrm{L}+3$ & $29.24 \%$ & & & & $\mathrm{H} \rightarrow \mathrm{L}+3$ & $27.92 \%$ \\
\hline & & & $\mathrm{H}-1 \rightarrow \mathrm{L}+3$ & $19.34 \%$ & & & & $\mathrm{H}-1 \rightarrow \mathrm{L}+3$ & $19.72 \%$ \\
\hline & & & $\mathrm{H}-1 \rightarrow \mathrm{L}+2$ & $7.43 \%$ & & & & $\mathrm{H}-1 \rightarrow \mathrm{L}+2$ & $6.01 \%$ \\
\hline & & & $\mathrm{H}-4 \rightarrow \mathrm{L}+4$ & $2.11 \%$ & & & & & \\
\hline & 552.67 & 0.0065 & $\mathrm{H}-4 \rightarrow \mathrm{L}+2$ & $38.56 \%$ & & 514.48 & 0.0021 & $\mathrm{H}-4 \rightarrow \mathrm{L}+2$ & $39.73 \%$ \\
\hline & & & $\mathrm{H}-1 \rightarrow \mathrm{L}+4$ & $26.73 \%$ & & & & $\mathrm{H}-1 \rightarrow \mathrm{L}+4$ & $25.71 \%$ \\
\hline & & & $\mathrm{H} \rightarrow \mathrm{L}+4$ & $25.74 \%$ & & & & $\mathrm{H} \rightarrow \mathrm{L}+4$ & $24.97 \%$ \\
\hline & & & $\mathrm{H}-4 \rightarrow \mathrm{L}+3$ & $4.40 \%$ & & & & $\mathrm{H}-4 \rightarrow \mathrm{L}+3$ & $4.50 \%$ \\
\hline & & & $\mathrm{H}-2 \rightarrow \mathrm{L}+4$ & $2.06 \%$ & & & & & \\
\hline & 552.18 & 0.0077 & $\mathrm{H}-3 \rightarrow \mathrm{L}+3$ & $39.35 \%$ & & 514.03 & 0.0023 & $\mathrm{H}-3 \rightarrow \mathrm{L}+3$ & $40.69 \%$ \\
\hline & & & $\mathrm{H} \rightarrow \mathrm{L}+5$ & $29.03 \%$ & & & & $\mathrm{H} \rightarrow \mathrm{L}+5$ & $27.96 \%$ \\
\hline & & & $\mathrm{H}-1 \rightarrow \mathrm{L}+5$ & $25.97 \%$ & & & & $\mathrm{H}-1 \rightarrow \mathrm{L}+5$ & $24.81 \%$ \\
\hline & & & $\mathrm{H}-3 \rightarrow \mathrm{L}+2$ & $4.66 \%$ & & & & $\mathrm{H}-3 \rightarrow \mathrm{L}+2$ & $4.86 \%$ \\
\hline & 540.9 & 0.0402 & $\mathrm{H}-2 \rightarrow \mathrm{L}+1$ & $33.23 \%$ & & 492.95 & 0.0282 & $\mathrm{H}-2 \rightarrow \mathrm{L}+1$ & $31.67 \%$ \\
\hline & & & $\mathrm{H}-5 \rightarrow \mathrm{L}$ & $25.71 \%$ & & & & $\mathrm{H}-5 \rightarrow \mathrm{L}$ & $26.49 \%$ \\
\hline & & & $\mathrm{H}-2 \rightarrow \mathrm{L}$ & $21.10 \%$ & & & & $\mathrm{H}-2 \rightarrow \mathrm{L}$ & $20.87 \%$ \\
\hline & & & $\mathrm{H}-5 \rightarrow \mathrm{L}+1$ & $15.44 \%$ & & & & $\mathrm{H}-5 \rightarrow \mathrm{L}+1$ & $16.15 \%$ \\
\hline
\end{tabular}

